\title{
Frühgeburtenrate in 6 Perinatalzentren in Baden-Württemberg - Potential zur Reduktion der Frühgeborenenzahl
}

\section{Preterm Birth Rate at 6 Centres for Perinatal Medicine in Baden- Württemberg - Potential to Reduce Prematurity}

\section{(C) (1) $\odot$}

\section{Autoren}

Andreas Trotter ${ }^{1}$, Ricarda Schreiber ${ }^{1}$, Silvia Sander ${ }^{2}$, Rainer Muche ${ }^{2}$, Wolfram Lucke ${ }^{3}$

Institute

1 Klinik für Kinder und Jugendliche, Singen

2 Institut für Epidemiologie und Medizinische Biometrie, Universität, Ulm

3 Perinatalzentrum, Singen

\section{Schlüsselwörter}

Schwangerschaft, Frühgeburt, Frühgeborenenrate, Frühgeborene, Ergebnisqualität, Perinatalzentren

\section{Key words}

pregnancy, preterm birth, preterm birth rate, preterm

infants, quality management, perinatal centre

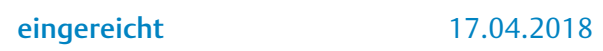

Korrespondenzadresse

Prof. Andreas Trotter, MD

Hegau-Bodensee-Klinikum

Klinik für Kinder und Jugendliche

Virchowstraße 10

78224 Singen

Tel.: +49/7731/89 2800, Fax: +49/7731/89 2805

andreas.trotter@web.de

\section{ZUSAMMENFASSUNG}

Hintergrund Zur Qualitätssicherung der Versorgung Früh- und Reifgeborener wurden vom Gemeinsamen Bundesausschuss umfangreiche Struktur- und Personalvorgaben formuliert. Die Ergebnisqualität für das neonatale Outcome wird bereits seit langer Zeit erhoben. Obwohl Deutschland einer der höchsten Frühgeburtenraten in Europa aufweist, existieren bislang keine zentrumsspezifischen Daten zur Frühgeburtenrate.
Methoden An 6 Perinatalzentren Level 1 in Baden-Württemberg wurden über 2 Jahre alle Schwangeren erfasst, die mit einem Schwangerschaftsalter zwischen $22+0$ und $31+6$ Wochen in der Frauenklinik aufgenommen wurden $(n=2972)$. Sofern sie innerhalb von $24 \mathrm{~h}$ nach Aufnahme mindestens eines der folgenden sekundären Einschlusskriterien 1. Gesicherte Amnionruptur, 2. Zervixlänge $<25$ mm oder 3. Tokogramm mit $>3$ Wehen von je mind. $30 \mathrm{sec}$. Dauer in 30 min erfüllten, wurde die Frühgeburtenrate vor $32+0$ Schwangerschaftswochen bestimmt. Für 70,6\% der Frühgeborenen konnte das neonatale Outcome erhoben werden.

Ergebnis Bei $21,1 \%(n=279)$ der Schwangeren, die mindestens ein sekundäres Einschlusskriterium erfüllten ( $n=1325)$, kam es vor $32+0$ SSW zur Geburt von 344 Frühgeborenen. Die Frühgeburtenrate lag zwischen 16,8 und 27,9\% (Differenz $11,1 \%)$. Eine niedrigere Frühgeburtenrate war nicht mit einem schlechteren neonatalen Outcome vergesellschaftet.

Schlussfolgerung Um die Ergebnisqualität eines Perinatalzentrums beurteilen zu können, sollte die Frühgeburtenrate als Qualitätsindikator Einzug finden. Eine Reduktion der Frühgeburtenrate um $5 \%$ könnte die Zahl der Frühgeborenen < $1500 \mathrm{~g}$ Geburtsgewicht in Deutschland um 2400/Jahr (ca. 25\%) reduzieren.

\section{ABSTRACT}

Background The Federal Joint Committee of Germany defined structural and staff requirements for the care of preterm and term infants as a proxy measure for quality. Neonatal outcome has been evaluated as a quality marker for a long time. While Germany has one of the highest preterm birth rates in Europe, no data exist on centre-specific preterm birth rates.

Methods Over 2 years, all pregnant women admitted to six centres for perinatal medicine in Baden-Württemberg at a gestational age between $22+0$ and $31+6$ weeks were eligible $(n=2972)$. The preterm birth rate before $32 \mathrm{wks}$ of gestation was evaluated if at least one of the following secondary inclusion criteria was present within $24 \mathrm{~h}$ after admission: 1) premature rupture of membranes, 2) cervical length less than $25 \mathrm{~mm}$, or 3 ) more than 3 contractions of at least 30 -s duration within a 30 -min period. Neonatal outcome could be documented for $70.6 \%$ of infants. 
Results Of the women who met at least one secondary inclusion criterion ( $n=1325), 21.1 \%(n=279)$ delivered 344 preterm infants before 32 weeks. The preterm birth rate was between 16.8 and $27.9 \%$ (11.1\% difference). A lower preterm birth rate was not associated with a worse neonatal outcome.
Conclusion Preterm birth rate should become a quality indicator for perinatal care. A reduction of the preterm birth rate of $5 \%$ could help to reduce the number of preterm infants by up to 2,400 per year in Germany (about 25\%).

\section{Einleitung}

Erstmals im Jahr 2005 wurden vom Gemeinsamen Bundesausschusses (G-BA) umfangreiche strukturelle Voraussetzungen als Maßnahme zur Qualitätsverbesserung für die Versorgung von Frühgeborenen in Deutschland beschlossen [1]. Vier Jahre später wurde eine Regelmäßigkeitszahl [2] und schließlich eine Mindestmenge von 14 zu versorgenden Frühgeborenen/Jahr und Perinatalzentrum (PNZ) mit einem Geburtsgewicht (GG) unter $1250 \mathrm{~g}$ festgelegt [3]. Die Mindestmenge wurde ein Jahr später von 14 auf 30 erhöht [4]. Nach Klagen mehrerer Kliniken gegen die Mindestmengenerhöhung wies das Bundessozialgericht die Erhöhung mit der Begründung ab [5], dass die Studienlage nicht uneingeschränkt die Einschätzung rechtfertigt, dass die Güte der Versorgung Frühgeborener durch eine Erhöhung der Mindestmenge in relevanter Weise zusätzlich gefördert werden kann.

Vom G-BA wurde auch festgelegt, dass Daten zur frühen und späten Ergebnisqualität bei der neonatologischen Versorgung veröffentlicht werden müssen (z. B. Mortalität, Rate an Hirnblutungen usw.). Die Ergebnisdaten für jedes PNZ sind seither frei für jedermann zugänglich unter www.perinatalzentren.org. Bislang gibt es keinerlei G-BA Vorgaben zur Erhebung der pränatalen Behandlungsqualität im Hinblick auf die Frühgeburtenrate, was auch von dem unparteiischen Vorsitzenden Herrn Prof. Hecken bereits öffentlich als Schwachstelle benannt wurde. Die Frühgeburtenraten in den PNZ sind nicht bekannt jedoch vermutlich ein entscheidendes aber bislang nicht beachtetes Qualitätskriterium für ein PNZ, da Frühgeburtlichkeit mit einer wesentlichen Mortalität und Morbidität assoziiert ist. Deutschland hat mit 8,6\% in 2016 [6] eine der höchsten Frühgeburtenraten in Europa [7].

Die Studie soll Aufschluss darüber geben, wie hoch die Frühgeburtenrate bei einem definierten Kollektiv von Schwangeren ist und wie stark sie zwischen verschiedenen PNZ streut. Entsprechende Daten aus der externen vergleichenden Qualitätssicherung nach $\S 137$ SGB V Modul Geburtshilfe stehen nicht zur Verfügung. Des Weiteren soll geprüft werden, ob ein Zusammenhang zwischen der Frühgeburtenrate mit dem Outcome der Frühgeborenen besteht.

\section{Patienten und Methoden}

Die Frühgeburtenrate und das Outcome der Frühgeborenen sollte im Routinebetrieb an 6 PNZ im Rahmen einer prospektiven Beobachtungsstudie mit retrospektivem Anteil erhoben werden und den Ist-Zustand zwischen den 6 PNZ darstellen. Die Datenerhebung wurde von Juli 2014 bis Juni 2016 an 6 PNZ des QuMiK-Klinikverbundes (Qualität und Management im Krankenhaus: Partnerschaft von Krankenhäusern, Kliniken und Gesundheitseinrichtungen in kommunaler Trägerschaft in Baden-Württemberg, www.qumik. de) durchgeführt. Alle 6 PNZ erfüllen die Strukturvorgaben für ein
PNZ Level I und versorgen pro Jahr zwischen 34 und 55 Frühgeborene $<1500 \mathrm{~g}$ Geburtsgewicht (Mittelwert 44,3 Frühgeborene; Quelle www.perinatalzentren.org, 2016). Alle, aus welchen Gründen auch immer, stationär in den Frauenkliniken aufgenommenen Schwangeren mit einer Schwangerschaftsdauer zwischen $22+0$ und $31+6$ Wochen wurden erfasst (primäres Einschlusskriterium). Die Gestationsalterbestimmung erfolgte primär nach der NägeleRegel anhand der letzten Periode und wurde routinemäßig mittels biometrischer sonographischer Parameter (Scheitel-Steiss-Länge, biparietaler Durchmesser) in der Frühschwangerschaft nachkorrigiert (Mutterschaftsrichtlinie). Um aus den stationär aufgenommenen Schwangeren das Kollektiv mit einem erhöhten Risiko für eine Frühgeburt innerhalb verschiedener PNZ gegenüberstellen zu können, wurden sekundäre Einschlußkriterien definiert: 1. Gesicherte Amnionruptur (sichtbarer Abgang von Fruchtwasser mit Vernixanteilen und/oder immunologischer Nachweis) [8, 9] und/oder 2. Zervixlänge vaginalsonographisch $<25 \mathrm{~mm}$ [10]und/oder 3. Tokogramm mit>3 Wehen von je mind. 30 s Dauer in 30 min (in Anlehnung an die Zeitvorgaben in der AWMF Leitlinie 015/036 der DGGG und im Konsens der 6 PNZ). Sofern mindestens ein sekundäres Einschlusskriterium innerhalb der ersten 24 Stunden nach Aufnahme erfüllt war, wurde der weitere Schwangerschaftsverlauf bis zum Erreichen von 31+6 Schwangerschaftswochen (SSW) dokumentiert. Um eine möglichst große Akzeptanz zur Teilnahme an der Studie im Routinebetrieb zu erreichen, wurde bewusst auf die Erhebung weiterer bekannter Einflussgrößen (wie z. B. Zahl vorangehender Schwangerschaften, Modus vorangehender Geburten, Diabetes, Präeklampsie, HELLP-Syndrom, Amnioninfektion, pathologischer Doppler usw.) verzichtet und nur die 3 oben erwähnten Einschlusskriterien erfasst. Eine multifaktorielle Adjustierung der Ergebnisse ist größer angelegten Studien vorbehalten. Entsprechend dem Votum der Ethikkommission war die Erhebung des Geburtsdatums bei pseudonymisierter Erfassung ohne vorliegende Einwilligung der Schwangeren zu unterlassen. Wurde vor $32+0$ SSW mindestens ein Frühgeborenes lebend geboren, erfolgte die Aushändigung der Patienteninformation und der Einwilligungserklärung an die Sorgeberechtigten. Im Falle einer Zustimmung wurde das postnatale Outcome des Frühgeborenen erfasst. Neben der Mortalität wurden folgende vier Morbiditäten bei Entlassung nach Hause erhoben: intraventrikuläre Hämorrhagie (IVH) $>\mathrm{II}^{\circ}$, zusätzlicher Sauerstoffbedarf bei Entlassung, Operation einer nekrotisierenden Enterokolitis (NEC) und Therapie einer Retinopathie. Wenn mindestens eine dieser 4 Morbiditäten vorlag, wurde das Vorliegen einer kombinierten Morbidität dokumentiert. Die Daten von Mutter und Kind wurden pseudonymisiert erfasst.

Um eine möglichst hohe Repräsentativität der Datenerhebung sicherzustellen, wurde vom Controlling der einzelnen Studienhäuser die Anzahl an Frauen mit den ICD-Kodierungen O 09.3 (Schwan- 
gerschaftsdauer 20-25 vollendete Wochen) und O 09.4 (26-33) nach dem ersten Studienjahr abgefragt. Mit den Ergebnisslisten wurde durch einen unabhängigen externen Gutachter (F. Pohlandt, Ulm) durch 20 Stichproben die Vollständigkeit der Erhebung evaluiert. Da in allen PNZ die Erhebungsrate unter $90 \%$ lag, wurde beschlossen, am Ende des gesamten Studienzeitraums eine umfängliche Nacherhebung vorzunehmen. Von 4041 in dem 2 Jahreszeitraum stationär aufgenommenen Schwangeren mit den genannten ICD-Codes erfüllten 3067 das primäre Einschlusskriterium (22+0 bis $31+6$ SSW). Für die Bearbeitung der Stichproben und die pseudonymisierte Nacherhebung vor Ort wurde jeweils eine Verpflichtung auf das Datengeheimnis der jeweiligen Klinik entsprechend den Vorgaben des Datenschutzbeauftragten des QuMiK-Verbundes gegengezeichnet. Nach Abschluss der Nacherhebung konnten insgesamt 2972 von 3067 Schwangeren erfasst werden (97\%). Die Studie wurde von der Ethikkommission der Landesärztekammer Baden-Württemberg bewilligt (Az F-2014-003).

\section{Statistik}

Die erhobenen Daten wurden in einem ersten Auswertungsschritt deskriptiv statistisch ausgewertet. Für qualitative Merkmale wurden absolute und relative Häufigkeiten und für quantitative Merkmale Minimum, Median, Maximum sowie Mittelwert angegeben. Die Auswertungen werden für das Gesamtkollektiv sowie getrennt nach PNZ dargestellt, um Unterschiede zwischen den PNZ identifizieren zu können.

Der Einfluss der PNZ auf die Zielgröße Eintreten einer Frühgeburt (ja vs. nein) wurde mit univariater und multipler logistischer Regression überprüft. Die Schätzung des Effekts der PNZ erfolgt dabei dummy-kodiert, PNZ 5 als PNZ mit der geringsten Frühgeburtenrate wurde immer als Referenzkategorie verwendet [11]. Da nur 3 wichtige Einflussgrößen erhoben wurden, konnte für einen Vergleich zwischen den PNZ nur der Einfluss bzgl. der erhobenen Einflussgrößen Amnionruptur (ja vs. nein), Zervixlänge $<25 \mathrm{~mm}$ (ja vs. nein) und Tokogramm > 3 Wehen (ja vs. nein) auf die Zielgröße Eintreten einer Frühgeburt adjustiert werden [12]. Die Zielgröße kombinierte Morbidität (ja vs. nein) des Kindes wurde nur univariat geprüft. Angegeben werden rohe bzw. adjustierte Odds Ratios (OR) mit zugehörigen $95 \%$-Konfidenzintervallen (KI) und orientierenden $\mathrm{p}$-Werten.

Um einen Zusammenhang zwischen Frühgeburtenrate und kombinierter Morbidität untersuchen zu können, werden diese Kenngrößen grafisch in einer bivariaten Verteilung für die PNZ gegenübergestellt und der Korrelationskoeffizient (Spearmans R) bestimmt.

\section{Ergebnisse}

\section{Frühgeburtenrate bei Schwangeren mit $22+0$ bis $31+6$ SSW}

In dem Untersuchungszeitraum von 2 Jahren wurden 2972 Schwangere erfasst ( Abb. 1). Davon erfüllten 1363 Schwangere (45,9\%) mindestens ein sekundäres Einschlusskriterium (40,6-53,5\% je PNZ, - Tab. 1). Die stationäre Aufnahme erfolgte im Mittel bei $28+2$ SSW und variierte zwischen den PNZ von $28+1$ bis $29+0$ (Mittelwerte). Bei 6 Schwangeren kam es vor $32+0$ SSW zu einer
Totgeburt, 32 Schwangere wurden vor $32+0$ SSW verlegt. Damit standen 1325 Schwangere für die weitere Analyse zur Verfügung. Die Frühgeburtenrate vor $32+0$ SSW lag insgesamt bei $21,1 \%$ (279/1325) und variierte zwischen den PNZ von 16,8-27,9\% ( $p=0,073$, univariate Analyse). Die Zahl der Frühgeburten vor $32+0$ SSW bei Schwangeren, die mindestens ein sekundäres Einschlusskriterium erfüllten, wurde in Beziehung gesetzt zur durchschnittlichen Gesamtgeburtenzahl eines PNZ während des Studienzeitraums (2014-2016, - Tab. 1). Dabei zeigt sich ein Zusammenhang zwischen Frühgeburtenrate und dem Anteil von Frühgeburten an der Gesamtgeburtenzahl $\left(r_{s}=0,85\right)$. Die 279 Schwangeren haben insgesamt 344 lebende Kinder entbunden (1,23 Frühgeborene) Frühgeburt). Vergleicht man das PNZ 5 (Referenz, niedrigste Frühgeburtenrate) mit den anderen PNZ, so ist die OR für eine Frühgeburt vor 32 + 0 SSW im PNZ 4 mit 1,9 [95\% KI 1,1-3,3] und im PNZ 6 mit 1,7 [1,04-2,7] höher (• Abb. 2, schwarze Balken).

In $>$ Tab. 2 werden die 3 wichtigsten Risikofaktoren für eine Frühgeburt vor $32+0$ SSW zwischen den PNZ betrachtet. War als sekundäres Einschlusskriterium „Amnionruptur“ erfüllt, zeigte sich insgesamt eine Frühgeburtenrate von 77,3\% (126/163). Im PNZ 6 führte eine Amnionruptur in 96,3\% zur Frühgeburt vor 32 + 0 SSW während im PNZ 5 die Rate nur bei 59,1\% lag.

Traf als sekundäres Einschlusskriterium „Zervixlänge $<25 \mathrm{~mm}$ “ zu, zeigte sich insgesamt eine Frühgeburtenrate von 20,1\% (193/960) mit einer Variation in den 6 PNZs von 15,6\% (PNZ3) und 26,3\% (PNZ 4). War als sekundäres Einschlusskriterium „Tokogramm > 3 Wehen“ erfüllt, lag die Frühgeburtenrate insgesamt bei 24,4\% (155/634). Im PNZ 1 führte ein Tokogramm > 3 Wehen in nur 20,3\% zur Frühgeburt während im PNZ 4 die Rate bei 30,7 \% lag. Bei 37 von 1325 Schwangeren (2,8\%) waren alle 3 sekundären Einschlusskriterien erfüllt. Die Frühgeburtenrate lag dann bei insgesamt 91,9\% (34/37) mit einer Variation in den 6 PNZs von 71,4\% (PNZ 5), 85,7\% (PNZ 4) und $100 \%$ (PNZ 1,2,3,6).

Nach Adjustierung des PNZ-Einflusses zeigt sich im multiplen logistischen Regressionsmodell für die 3 sekundären Einschlusskriterien auf die Zielgröße Eintreten einer Frühgeburt vor $32+0$ SSW für die „Zervixlänge $<25$ mm“ eine OR von 3,1 [2,0-4,7] $(p<0,001)$ und für „Tokogramm $>3$ Wehen“ eine OR von 3,6 [2,5-5,2] $(p<0,001)$. Extrem hoch zeigt sich die OR mit 56,5 [33,2-96,2] beim Vorhandensein des Einschlusskriteriums „Amnionruptur“ $(p<0,001)$. Für die PNZ zeigt sich nach Adjustierung bezüglich der 3 sekundären Einschlusskriterien ein ähnliches Bild wie in der univariaten Auswertung (s. o.) für das Eintreten einer Frühgeburt vor $32+0$ SSW $(p=0,108)$. Die OR für eine Frühgeburt vor $32+0$ SSW zeigt ein etwa doppelt so hohes Risiko für PNZ 4 (OR = 1,9 [1,004$3,7])$ und PNZ $6(O R=2,0[1,1-3,7])$ im Vergleich zum ReferenzPNZ 5 mit der niedrigsten Frühgeburtenrate ( $\mathbf{A} \mathbf{A b}$. 2, blaue Balken).

\section{Outcome der Frühgeborenen}

Von den 279 Entbundenen stimmten 197 (70,6\%) der Erhebung des postnatalen Outcome zu. Der Anteil an erzielten Einwilligungen in den 6 PNZ lag zwischen 55,3 und 90,3\%. Die 197 schwangeren Frauen mit Zustimmung haben 253 Kinder bei im Mittel $28+4$ SSW mit einem GG von $1193 \mathrm{~g}$ entbunden (1,28 Frühgeborene/Frühgeburt) ( $>$ Tab. 3), 199 Kinder (78,7\%) wiesen ein GG von<1500 g (152 Frühgeburten) bzw. 147 (58,1 \%) ein GG <1250 g 


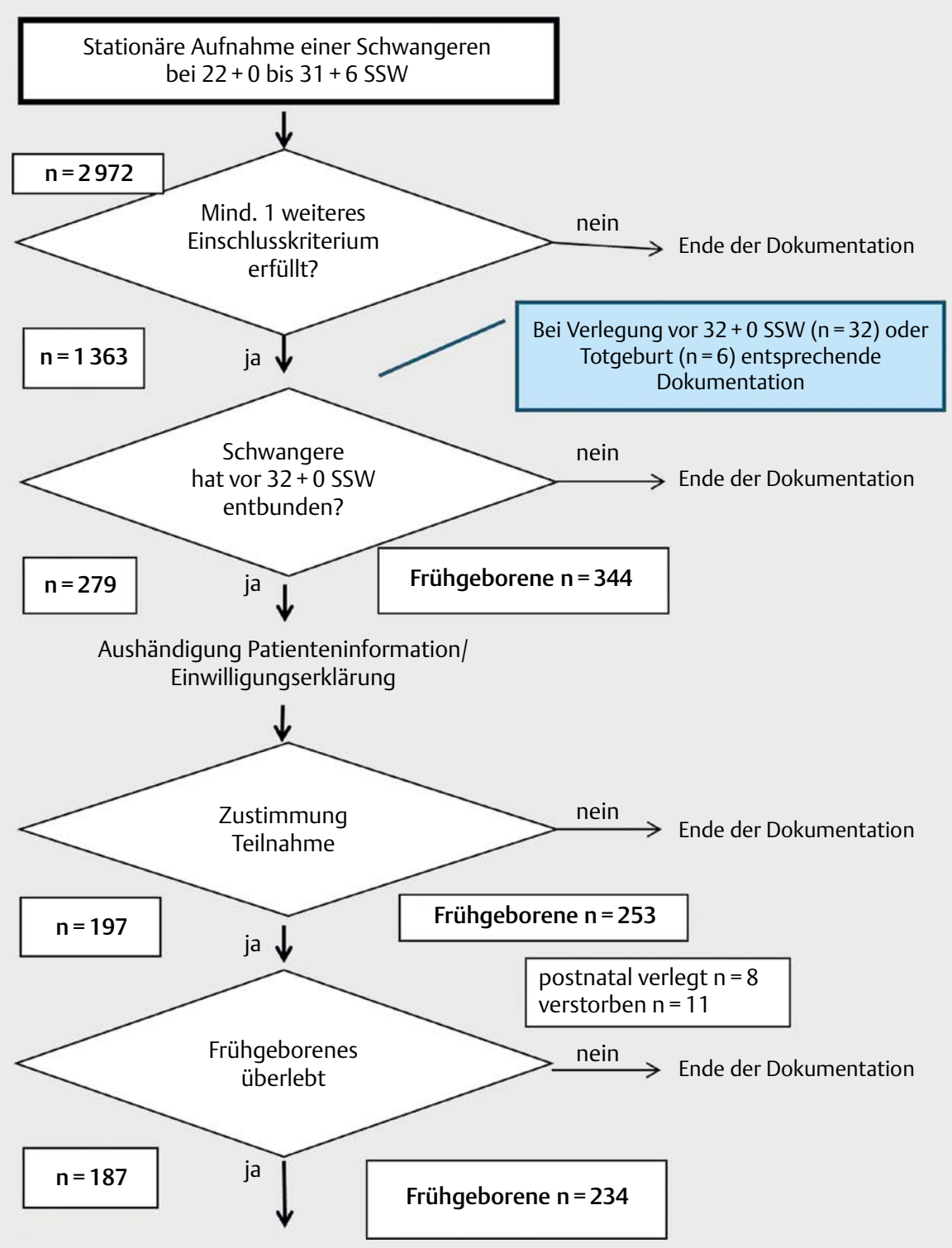

Ende der Dokumentation

Abb. 1 Studienablauf mit Fallzahlen.

(112 Frühgeburten) auf. Die Relation Frühgeborene/Frühgeburt ist für diese beiden Gewichtsklassen gleich (<1500 g: 1,31;<1250 g: 1,31). Von den 253 Frühgeborenen mit Einwilligung zur Erhebung des Outcomes wurden 8 postnatal verlegt, 11 Frühgeborene sind postnatal verstorben (4,5\%, davon 4 palliativ versorgt, > Tab. 3 ). Das Outcome der 8 verlegten Frühgeborenen konnte nicht erhoben werden. Selbst wenn man die 8 postnatal verlegten Frühgeborenen als gestorbene werten würde, läge die Mortalität bei maximal 7,5\%. Somit kann von 234/253 (92,5\%) überlebenden Frühgeborenen die kombinierte Morbidität betrachtet werden ( $\triangleright$ Tab. 3). Insgesamt wiesen 35 Frühgeborene (15\%) mindestens eine Morbidität bei Entlassung auf ( 6 Frühgeborene eine, 25 zwei, 4 drei und keines alle 4 Morbiditäten).
In $>$ Abb. $\mathbf{3}$ zeigt sich zwischen der Frühgeburtenrate und der kombinierten Morbidität für die 6 PNZ kein Zusammenhang $\left(r_{s}=0,03\right)$. Auffällig ist PNZ 4 mit der höchsten Frühgeburtenrate bei gleichzeitig niedrigster kombinierter Morbidität. Die überlebenden Frühgeborenen von PNZ 4 hatten neben der höchsten Frühgeburtenrate auch das höchste SS-Alter und GG bei Entbindung.

\section{Diskussion}

Bei etwa einem Fünftel aller Schwangeren $(21,1 \%)$ mit sekundären Einschlusskriterien kam es zu einer Frühgeburt vor 32 + 0 SSW. Dabei zeigten sich zwischen 6 PNZ Level 1 in Baden-Württemberg deutliche Unterschiede bei der Erfolgsrate, eine Frühgeburt zu ver- 


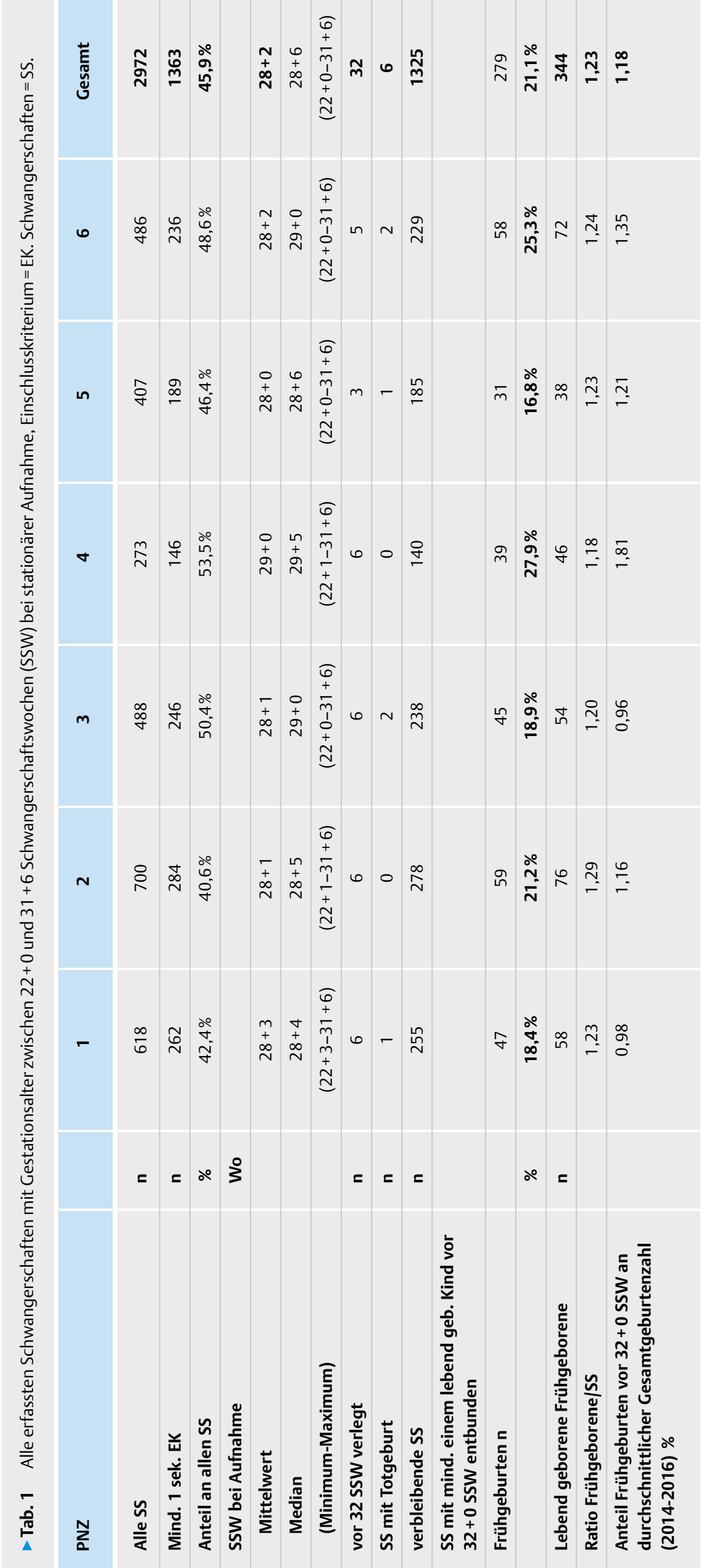


hindern. Zieht man das PNZ, dass die niedrigste Frühgeburtenrate aufwies, als Referenz heran, werden die Unterschiede sowohl in der univariaten als auch in der multiplen logistischen Regression auffällig. Zwei PNZ (4 und 6) wiesen eine doppelt so hohe Frühgeburtenrate auf. Das Vorliegen einer Amnionruptur war der stärkste Prädiktor für das Auftreten einer Frühgeburt. Die Mortalität der Frühgeborenen lag mit 4,5\% im internationalen Vergleich mit ähnlichem Kollektiv (in 6 europäischen Ländern 8,0-15,7\%; [13]) führend niedrig. Weshalb PNZ 4 bei höchster Frühgeburtenrate die niedrigste Morbidität aufwies, lässt sich vermutlich nicht nur auf das gleichzeitg vorhandene höchste SS-Alter und GG bei Entbindung zurückführen, sondern auch auf weitere Einflussgrößen, die nicht erhoben wurden.

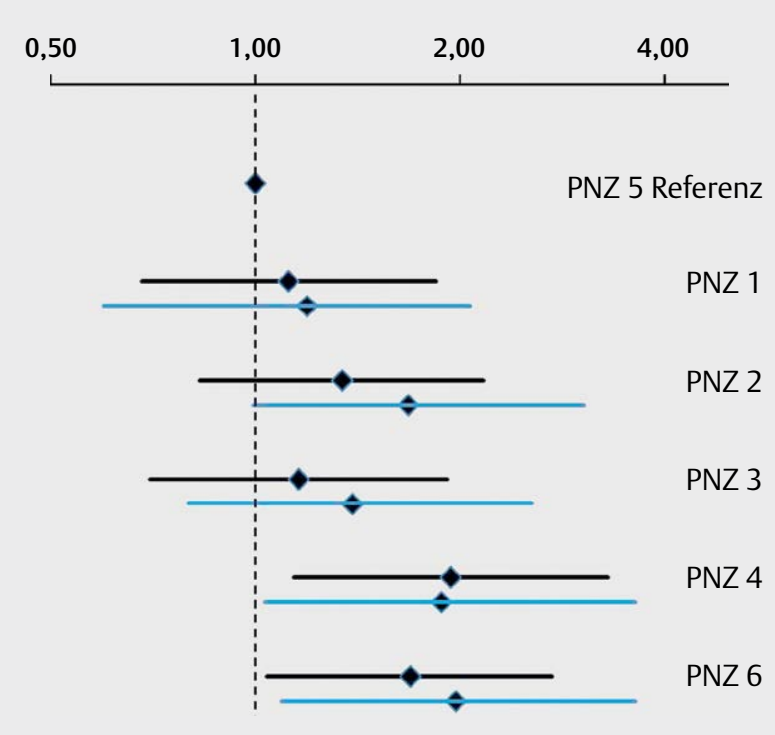

- Abb. 2 Vergleich der Frühgeburtenrate zwischen den PNZ (PNZ 5, Referenz - niedrigste Frühgeburtenrate). Rohe (schwarze Balken) und adjustierte (blaue Balken, adjustiert für Amnionruptur, Zervixlänge $<25$ mm und Tokogramm $>3$ Wehen) OR mit 95\% Konfidenzintervall.
Aus diesen Daten leitet sich die Forderung ab, bei der Beurteilung der Versorgungsqualität eines PNZ die Frühgeburtenrate als Qualitätsindikator einzuführen. Wenn wie in unserer Erhebung die Frühgeburtenrate zwischen den PNZ zwischen 16,8 und 27,9\% variiert, wäre es wichtig, diesen Indikator künftig zur transparenten Darstellung der Ergebnisqualität eines PNZ darzustellen.

Im Jahr 2016 wurden in Deutschland in 215 Perinatalzentren (161 Level I, 54 Level II) 9284 Frühgeborene mit GG < $1500 \mathrm{~g}$ (8706 Level I, 578 in Level II) versorgt (Quelle: www.perinatalzentren.org). Bei einem Verhältnis Frühgeborenen/Frühgeburt von 1,31 (s.o.) und einer angenommenen Frühgeburtenrate von $20 \%$ (21,1 \% in dieser Studie) würden 9284 Frühgeborene etwa 36000 Risikoschwangeren entsprechen. Wenn durch eine optimierte Therapie die Frühgeburtenrate in Deutschland um 5 \% gesenkt werden könnte, was angesichts der Ergbnisse dieser Studie realistisch scheint (Frühgeburtenrate zwischen 16,8-27,9\%, Differenz 11,1\%), würde sich die Zahl der Frühgeburten um 1800/Jahr und damit die Zahl der Frühgeborenen < 1500 g GG um ca. 2400 (ca. 25 \%) reduzieren. In unserer Studie ergaben sich keine Hinweise, dass eine niedrigere Frühgeburtenrate mit einer höheren Morbidität der Frühgeborenen vergesellschaftet ist.

Die Erhebung an 6 PNZ in Baden-Württemberg liefert deskriptive Daten. Repräsentative $97 \%$ der Schwangeren, die zwischen $22+0$ und $31+6$ SSW stationär aufgenommen wurden und mindestens ein sekundäres Einschlusskriterium erfüllten, konnten ausgewertet werden, während mangels nicht vorliegender Einwilligung nur für 70,6\% der Frühgeborenen das postnatale Outcome erhoben werden konnte. Weitere Risikofaktoren für eine Frühgeburt wie im Methodenteil genannt sowie kindliche Risikofaktoren (z. B. fetale Wachstumsretardierung, Geschlecht, Fehlbildungen usw.) wurden nicht erhoben und konnten somit bei der Auswertung nicht berücksichtigt werden. Die Ergebnisse haben somit hypothesengenerierenden Charakter. Bekannte Risikofaktoren waren in einer internationalen Studie mit über 4 Mio. Einlingsgeburten mit 1/3 der Frühgeburten assoziiert, für weitere 2/3 müssen durch künftige Forschung weitere bisher nicht bekannte Risikofaktoren identifiziert werden [14].

- Tab. 2 Absolute (in Klammern) und relative Häufigkeiten von sekundären Einschlußkriterien und deren Assoziation mit einer Frühgeburt vor $32+0$ SSW von 1325 Schwangerschaften (SS), die mind. ein sekundäres Einschlusskriterium (EK) erfüllten, nicht verlegt wurden und nicht mit einer Totgeburt endeten.

\begin{tabular}{|c|c|c|c|c|c|c|c|}
\hline PNZ & 1 & 2 & 3 & 4 & 5 & 6 & Gesamt \\
\hline SS & 255 & 278 & 238 & 140 & 185 & 229 & 1325 \\
\hline Amnionruptur & $12,9 \%(33)$ & $11,2 \%(31)$ & $11,3 \%(27)$ & $16,4 \%(23)$ & $11,9 \%(22)$ & $11,8 \%(27)$ & $12,3 \%(163)$ \\
\hline Frühgeburtenrate & $75,8 \%(25)$ & $77,4 \%(24)$ & $81,5 \%(22)$ & $69,6 \%(16)$ & $59,1 \%(13)$ & $96,3 \%(26)$ & $77,3 \%(126)$ \\
\hline Zervixlänge < $25 \mathrm{~mm}$ & $62,7 \%(160)$ & $82,7 \%(230)$ & $78,2 \%(186)$ & $70,7 \%(100)$ & $75,1 \%(139)$ & $63,3 \%(145)$ & $72,4 \%(960)$ \\
\hline Frühgeburtenrate & $15,6 \%(25)$ & $21,3 \%(49)$ & $15,6 \%(29)$ & $26,0 \%(26)$ & $18,7 \%(26)$ & $26,2 \%(38)$ & $20,1 \%(193)$ \\
\hline Tokogramm auffällig & $\mathbf{5 4 , 1} \%(138)$ & $35,6 \%$ (99) & $39,1 \%(93)$ & $53,6 \%(75)$ & $47,0 \%(87)$ & $62,0 \%(142)$ & $47,8 \%(634)$ \\
\hline Frühgeburtenrate & $20,3 \%(28)$ & $29,3 \%(29)$ & $\mathbf{2 4 , 7} \%(23)$ & $30,7 \%(23)$ & $20,7 \%(18)$ & $23,9 \%(34)$ & $24,4 \%(155)$ \\
\hline Alle 3 EK gleichzeitig & $2,7 \%(7)$ & $2,5 \%(7)$ & $1,7 \%(4)$ & $5,0 \%(7)$ & $3,8 \%(7)$ & $2,2 \%(5)$ & $\mathbf{2 , 8} \%(37)$ \\
\hline Frühgeburtenrate & $100,0 \%(7)$ & $100,0 \%(7)$ & $100,0 \%(4)$ & $85,7 \%(6)$ & $71,4 \%(5)$ & $100,0 \%(5)$ & $\mathbf{9 1 , 9 \%}(34)$ \\
\hline
\end{tabular}




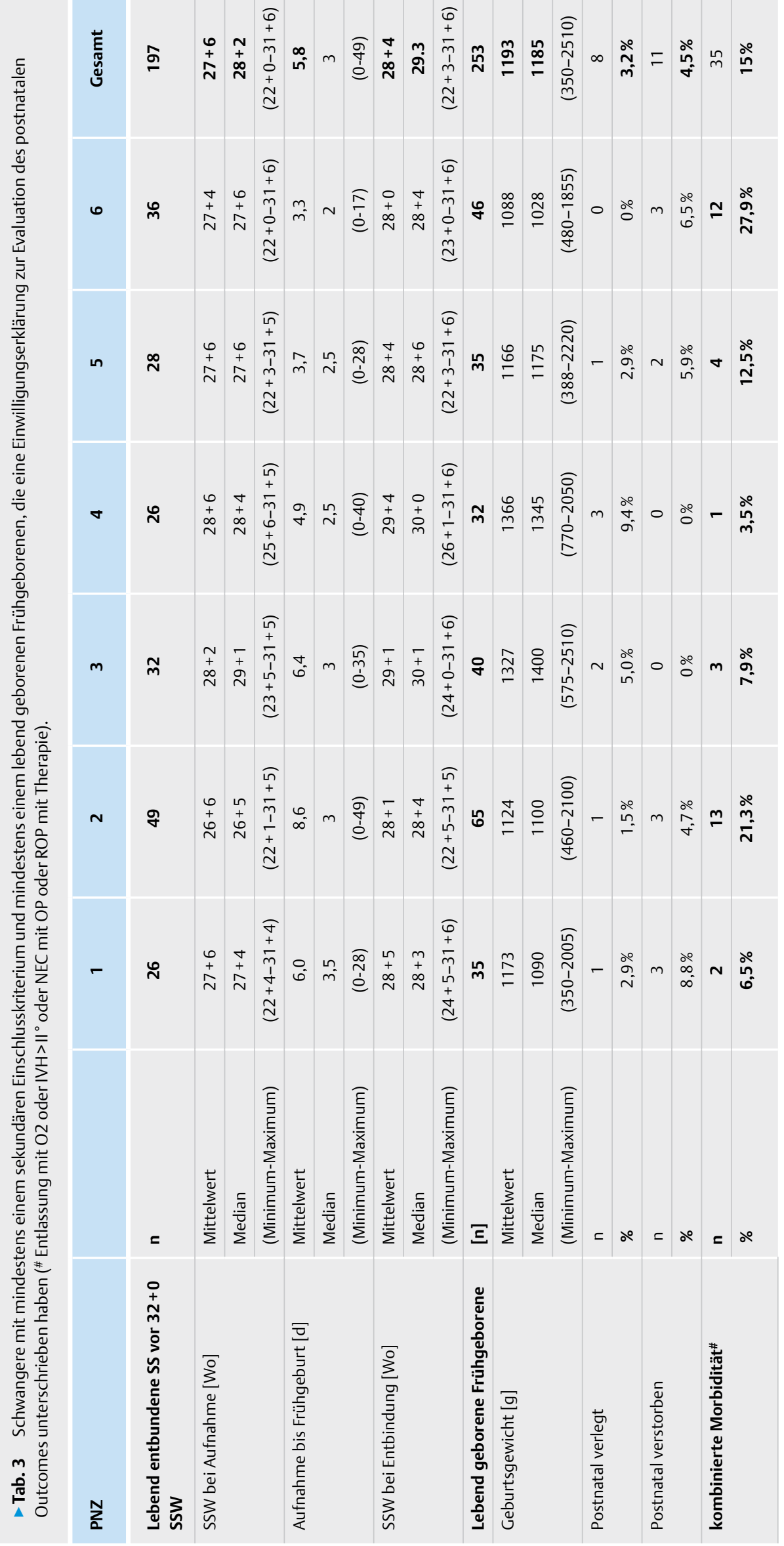




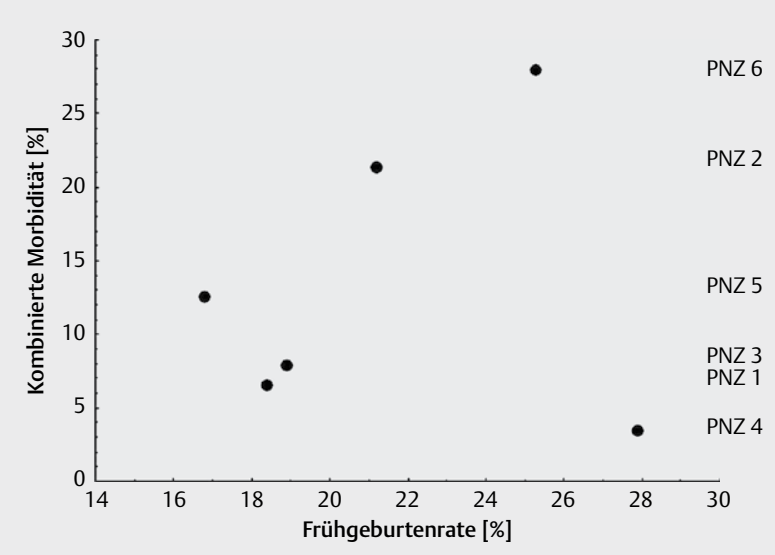

A Abb. 3 Gegenüberstellung der Frühgeburtenrate mit der kombinierten Morbidität der Frühgeborenen.

Die beträchtlichen Unterschiede in der Frühgeburtenrate vor 32 + 0 SSW für ein definiertes Risikokollektiv zwischen den 6 PNZ mit in der QFR-RL (Qualitätssicherungs-Richtlinie Früh- und Reifgeborene) festgelegten Struktur- und Personalvorgaben geben Anlass, sich über verschiedene Therapiestrategien bei der Verhinderung einer Frühgeburt auszutauschen, um in einer gemeinsamen Diskussion zu einer konsentierten und optimierten Therapie zur Verhinderung einer Frühgeburt zu kommen. Auch bei internationalen Vergleichen in 39 Ländern (alle mit sogenanntem Very High Human Developmental Index) finden sich deutliche Unterschiede in der Frühgeburtenrate für Frühgeborene<37 SSW (5,3-14,7\% bezogen auf alle Lebendgeborenen [7]). Dabei wurden 5 Interventionen identifiziert (Kein Rauchen währen der SS, Vermeidung multipler Embryotransfers, zervikale Zerklage, Progesteron-Supplementation und die Reduktion nicht medizinisch indizierter Sektios), die das Potenzial hätten, die Frühgeburtenrate um 5 \% zu senken. Wie von Rossi et al. [15] dargestellt, sind die Raten an Frühgeborenen mit $<2500$ und $<1500 \mathrm{~g}$ GG in Deutschland deutlich höher als in Schweden und Finnland. Eine Leitlinie, wie eine Frühgeburt am besten verhindert werden kann, existiert jedoch bislang nicht.

Aus den dargestellten Ergebnissen leiten sich gesundheitspolitisch wichtige Aspekte ab

An der Frühgeburtenrate und am neonatalen Outcome orientierte Umstrukturierungen haben größeres Potenzial, Frühgeburten zu verhindern und die Versorgung der Frühgeborenen noch weiter zu verbessern als Mindestmengen [16]. Der Anteil von Frühgeburten an der Gesamtgeburtenzahl könnte ein einfacher Surrogatparameter als Qualitätsindikator sein, berücksichtigt aber nicht regionale Unterschiede im Versorgungprofil eines PNZ von Schwangerschaften oberhalb von $32+0$ SSW. Vom G-BA erneut geplante Mindestmengenanhebungen für Frühgeborene liefern falsche Anreize und bergen das Risiko, mit hoher Qualität geburtshilflich und neonatologisch arbeitende PNZ zu verlieren. Eine gegenüber Schweden und Finnland (Länder mit hohem Zentralisierungrad bei der neonatologischen Versorgung) erhöhte Säuglingssterblichkeit in Deutschland [15] sollte dabei kein Argument sein, denn die Über- lebensrate Frühgeborener ist in Deutschland wie auch in unserer Erhebung dargestellt (s. o.) im internationalen Vergleich führend gut $[17,18]$. Nur etwa $9 \%$ der Variablität der Mortalität von Frühgeborenen < $1500 \mathrm{~g}$ erklären sich über die Fallzahl eines PNZ [19]. Geht man von einer Erhöhung auf 30 Frühgeborene $<1250 \mathrm{~g}$ für Level 1 PNZ aus, würden 70 der 161 PNZ Level 1 in Deutschland die Mindestmenge nicht erreichen (Herleitung im Detail im Anhang). Ein transparentes Beispiel, wie sich eine Mindestmengenerhöhung auswirken kann, ist im Anhang dargestellt.

\section{KERNAUSSAGEN}

- Die Frühgeburtenrate zeigte in 6 verschiedenen Perinatalzentren relevante Unterschiede.

- Die Frühgeburtenrate sollte bei der Beurteilung der Ergebnisqualität eines Perinatalzentrums als Qualitätsindikator Berücksichtigung finden.

- Eine Reduzierung der Frühgeburtenrate hat großes Potenzial die Zahl sehr kleiner Frühgeborener zu reduzieren.

- Es ergaben sich keine Hinweise, dass eine niedrigere Frühgeburtenrate mit einer höheren Morbidität der Frühgeborenen vergesellschaftet ist.

- Eine Leitlinie zur bestmöglichen Verhinderung von Frühgeburten wäre wünschenswert.

\section{Danksagung}

Wir danken

- allen Mitwirkenden Perinatalzentren für ihre wertvolle Beteiligung und Unterstützung bei der Durchführung der Studie:

- Klinikverbund Südwest, Kliniken Böblingen, Frauenklinik: Herr PD Dr. med. Weiss. Klinik für Kinder- und Jugendmedizin: Herr Prof. Dr. med. Teufel, Herrn Dr. Nachtrodt

- SLK-Kliniken Heilbronn GmbH, Klinikum am Gesundbrunnen, Frauenklinik: Herr Prof. Dr. med. Hackenberg, Klinik für Kinder- und Jugendmedizin: Herr Prof. Dr. med. Ruef

- Kliniken Ludwigsburg-Bietigheim gGmbH, Klinikum Ludwigsburg, Klinik für Frauenklinik und Geburtshilfe: Herr Prof. Dr. med. Heyl. Klinik für Kinder- und Jugendmedizin: Herr Dr. med. Walka

- Oberschwabenklinik GmbH, Krankenhaus St. Elisabeth, Frauenklinik: Frau Dr. med. Gropp-Meier. Kinder- und Jugendmedizin: Herr PD Dr. med. Artlich

- Schwarzwald-Baar Klinikum Villingen-Schwenningen $\mathrm{GmbH}$, Kliniken Villingen-Schwenningen, Herr Prof. Dr. med. Zieger. Klinik für Kinderheilkunde, Jugendmedizin und Kinderchirurgie: Herr PD Dr. med. Henschen

- Gesundheitsverbund Landkreis Konstanz, Hegau-Bodensee-Klinikum Singen - siehe Autoren

- Herrn Prof. Pohlandt, ehemals Universitätsklinikum Ulm, Sektion Neonatologie dem QuMiK-Klinikverbund für die Unterstützung 


\section{ANHANG}

Herleitung der Zahl von 70 PNZ, die eine Mindestmenge von 30 Frühgeborene $<1250 \mathrm{~g}$ GG nicht erreichen

Bei der zentralen Ergebnisveröffentlichung aller PNZ (Level 1) unter www.perinatalzentren.org wird nur die Zahl der Frühgeborenen unter $1500 \mathrm{~g} \mathrm{GG}$, die ein PNZ pro Jahr versorgt, aufgeführt. Um die Auswirkungen einer Erhöhung der Mindestmenge von Frühgeborenen mit GG $<1250 \mathrm{~g}$ auf z. B. 30 abschätzen zu können, muss deshalb die sich daraus ergebende Mindestmengen für Frühgeborenen mit $\mathrm{GG}<1500 \mathrm{~g}$ hochgerechnet werden.

Von den 253 Frühgeborenen mit Outcomedaten hatten 199 ein GG unter $1500 \mathrm{~g}$ und davon 147 (73,9\%) ein GG unter $1250 \mathrm{~g}$. Nach dem Jahresbericht 2016 der externen vergleichende Qualitätssicherung ( $\$ 137$ SGB V) in BadenWürttemberg wiesen 1394 Frühgeborene ein GG unter $1500 \mathrm{~g}$ und 969 ein GG unter $1250 \mathrm{~g}$ (entsprechend 69,5\%) auf, was sich mit unseren Ergebnissen deckt. Damit entspricht eine Zahl von 30 Frühgeborenen mit $<1250 \mathrm{~g} \mathrm{GG}$ (ca. $70 \%$ ) einer Zahl von ca. 43 Frühgeborenen (100\%) mit GG $1500 \mathrm{~g}$. Bei einer Erhöhung der Mindestmenge auf 30 Frühgeborene $<1250 \mathrm{~g}$ würden somit insgesamt 70 PNZ Level 1 die Mindestmenge nicht mehr erreichen (Stand Listung für 2016 unter www.perinatalzentren.org, für 2017 liegen die Daten noch nicht vor (09.04.18)).

\section{Beispiel, wie sich eine Mindestmengenerhöhung auf 30} Frühgeborene $<1250 \mathrm{~g}$ auswirken kann

Eine Mindestmenge von 30 Frühgeborenen mit GG $<1250 \mathrm{~g}$ entspricht nach unseren Schätzungen (1,31 Frühgeborene/ Frühgeburt, mittlere Entbindungsrate 21,1\%) 113 Risikoschwangeren. Wendet man die Frühgeburtenrate des als Referenz gewählten PNZ 5 an, würden 19 Schwangere entbinden (16,8\%, entsprechend 25 Frühgeborenen). Bei dem PNZ 4 mit der höchsten Frühgeburtenrate würden 32 Schwangere entbinden $(27,9 \%$, entprechend 41 Frühgeborenen).

\section{Interessenkonflikt}

Die Autoren geben an, dass kein Interessenkonflikt besteht.

\section{Literatur}

[1] BAnz Nr. 205 vom 28.10.2005

[2] BAnz Nr. 65 vom 30.04.2009

[3] BAnz Nr. 195 vom 12.2009

[4] BAnz Nr. 125 vom 18.08.2010

[5] 1. Senat, Az: B 1 KR 34/12 R

[6] IQTIG Qualitätsreport 2016 https://wwwiqtigorg/downloads/ ergebnisse/qualitaetsreport/IQTIG_Qualitaetsreport-2016pdf

[7] Chang $\mathrm{HH}$, Larson J, Blencowe $\mathrm{H}$ et al. Preventing preterm births: analysis of trends and potential reductions with interventions in 39 countries with very high human development index. Lancet 2013; 381 : 223-234

[8] Yu H, Wang X, Gao H et al. Perinatal outcomes of pregnancies complicated by preterm premature rupture of the membranes before 34 weeks of gestation in a tertiary center in China: A retrospective review. Biosci Trends 2015; 9: 35-41

[9] Mercer BM. Preterm premature rupture of the membranes: current approaches to evaluation and management. Obstet Gynecol Clin North Am 2005; 32: 411-428

[10] Crane JM, Hutchens D. Transvaginal sonographic measurement of cervical length to predict preterm birth in asymptomatic women at increased risk: a systematic review. Ultrasound Obstet Gynecol 2008; 31: 579-587

[11] Hosmer D, Lemeshow S. Applied logistic regression. $2^{\text {nd }}$. Aufl. New York: John Wiley Sons Inc; 2000

[12] Becker A, Stausberg J, Fischer B et al. Risikoadjustierung von Qualitätsindikatoren. Das Krankenhaus 2016; 11: 954-963

[13] Numerato D, Fattore G, Tediosi F et al. Mortality and Length of Stay of Very Low Birth Weight and Very Preterm Infants: A EuroHOPE Study. PLoS One 2015; 10: e0131685

[14] Ferrero DM, Larson J, Jacobsson B et al. Cross-Country Individual Participant Analysis of 4.1 Million Singleton Births in 5 Countries with Very High Human Development Index Confirms Known Associations but Provides No Biologic Explanation for 2/3 of All Preterm Births. PLoS One 2016; 11: e0162506

[15] Rossi R, Poets CF, Jorch G. Perinatalmedizinische Versorgung: Maximale Sicherheit für Mutter und Kind anstreben. DÄB 2015; 112: $18-20$

[16] Rochow N, Landau-Crangle E, Lee $S$ et al. Quality Indicators but Not Admission Volumes of Neonatal Intensive Care Units Are Effective in Reducing Mortality Rates of Preterm Infants. PLoS One 2016; 11: e0161030

[17] Trotter A, Pohlandt F. Versorgung sehr kleiner Frühgeborener in Deutschland international führend gut. Säuglingssterblichkeit ist ein ungeeigneter Surrogatparameter. Das Krankenhaus 2011; 3; 240-241

[18] Draper ES, Zeitlin J, Fenton AC et al. Investigating the variations in survival rates for very preterm infants in 10 European regions: the MOSAIC birth cohort. Arch Dis Child Fetal Neonatal Ed 2009; 94: F158-F163

[19] Rogowski JA, Horbar JD, Staiger DO et al. Indirect vs direct hospital quality indicators for very low-birth-weight infants. Jama 2004; 291: 202-209 